Case report

\title{
Magnetic resonance imaging as a clue to successful diagnosis of renal tuberculosis: a case report Nobuaki Matsui* and Tatsuo Morita
}

\author{
Address: Department of Urology, Jichi Medical University Hospital, 3311-1 Yakushiji, Shimotsuke, Tochigi, 3290498, Japan \\ Email: NM* - n-matsui@jichi.ac.jp; TM - moritatu@jichi.ac.jp \\ * Corresponding author
}

Received: 21 January 2009 Accepted: 3 August 2009 Published: 12 August 2009

Cases Journal 2009, 2:8879 doi: 10.4076/1757-1626-2-8879

This article is available from: http://casesjournal.com/casesjournal/article/view/8879

(C) 2009 Matsui and Morita; licensee Cases Network Ltd.

This is an Open Access article distributed under the terms of the Creative Commons Attribution License (http://creativecommons.org/licenses/by/3.0),

which permits unrestricted use, distribution, and reproduction in any medium, provided the original work is properly cited.

\begin{abstract}
Computed tomography is considered as the imaging modality of choice in the diagnosis of genitourinary tuberculosis, while magnetic resonance imaging may provide some informative features corresponding to the pathologic stage of the disease. We herein present a case report where magnetic resonance imaging showed the informative features, and a clue to further examinations in focusing on renal tuberculosis.
\end{abstract}

\section{Case presentation}

A 53-year-old Japanese woman was referred to our institution for recurrent cystitis. Abdominal sonogram was initially performed, revealing the presence of a left upper pole cystic lesion in a hydronephrotic kidney and dilatation of the ipsilateral ureter. Subsequent CT examination, including both plain and contrast-enhanced images confirmed the presence of a cystic mass at upper pole of the left kidney, accompanied by thinning and enhancement of the renal parenchyma. The dimensions of the lesion were $40 \times 25 \mathrm{~mm}$. No renal calcifications were noted. Left ureteral dilatation was also seen, with thickening and enhancement of the ureteral wall (Figure 1). Based on the CT findings, differential diagnosis included chronic pyelonephritis, papillary necrosis, medullary sponge kidney, renal cell carcinoma, transitional cell carcinoma and renal tuberculosis were considered. We then performed MRI for further examination, which depicted the thinned parenchymal left kidney lesion, of low intensity on both $\mathrm{T} 1$ and $\mathrm{T} 2$-weighted images, when compared to the normal renal parenchyma, heterogeneously enhancing after gadolinium administration. In addition, there was a nodule in the parenchyma that was not enhanced by gadolinium administration with iso intensity on T1-weighted images and low intensity on T2weighted images (Figure 2). MR imaging findings indicated inflammation and fibrosis with a granuloma formation, therefore strongly suggesting renal tuberculosis. Based on our findings, further examinations were scheduled in focusing on urinary tract tuberculosis. Subsequent cystoscopy showed reddish and edematous mucosa around left ureteral orifice and biopsied specimen revealed a granuloma. Urine culture and urine polymerase chain reaction (PCR) were positive for mycobacterium tuberculosis. Her tuberculin skin test was positive, showing $48 \times 62 \mathrm{~mm}$ rubor with induration, whereas her chest radiograph showed no nodular lesion or cavity. Under the diagnosis of urinary tract tuberculosis, she received antitubercular medications with isoniazid, rifampicin, pyrazinamide and ethambutol for 9 months. Because of persistent pyuria and left kidney dysfunction, left nephroureterectomy was subsequently 


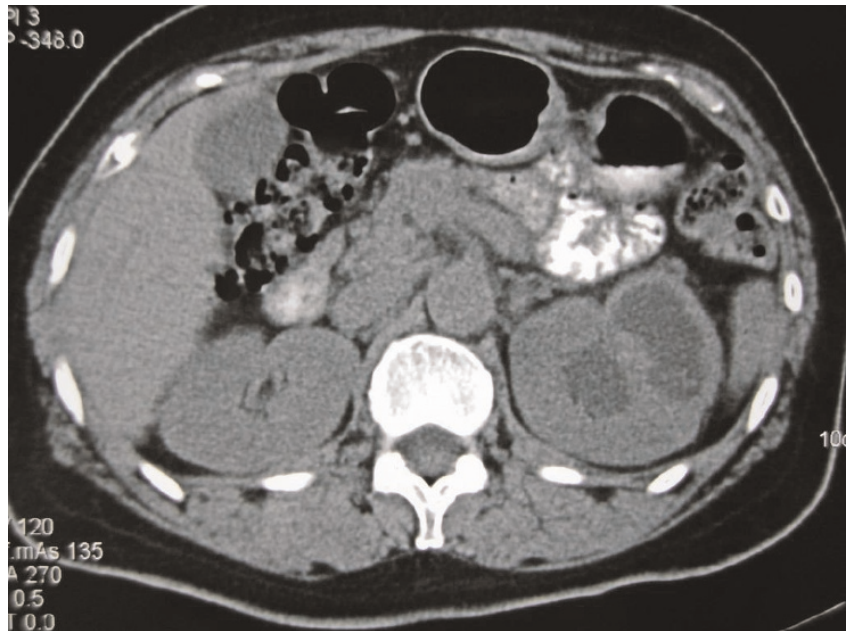

Figure I. Plain CT image demonstrates a cystic mass lesion in the upper pole of the left kidney. No calcifications were detected.

performed, although urine culture and PCR had become negative for mycobacterium tuberculosis. Pathological examinations of resected specimen revealed caseous necrotic granuloma and lymphocytes infiltration to the renal parenchyma and ureter, consisting with urinary tract tuberculosis. The patient is now well, without signs of disease recurrence five years after surgery.

\section{Discussion}

The genitourinary tract is one of the most common sites of extrapulmonary tuberculosis, accounting for $15-20 \%$ of infections outside lungs and approximately $4-8 \%$ with pulmonary tuberculosis will develop significant genitourinary tuberculosis [1]. The diagnosis is made on basis of urine culture studies or histopathologic analysis and is supported by radiologic studies. The findings of the disease on plain abdominal radiography are calcifications. Renal calcifications are a one of the common manifestations of tuberculosis at conventional radiography, occurring in 24-44\% [2]. Intravenous urography may show a variety of findings, including moth-eaten calyces, amputated infundibula, hydronephrosis or hydronephroureter due to ureteral strictures and non-function of a kidney $[3,4]$. CT is the most sensitive modality for renal calcifications which occur in over $50 \%$ of cases of genitourinary tuberculosis $[2,5]$ and is thought to be a mainstay in the diagnostic images of renal tuberculosis, showing renal parenchymal cavity, mass and scaring, local parenchymal thinning, stricture of infundibula $[4,6]$. CT urography is a relatively new imaging examination which can provide comprehensive evaluation of both renal parenchyma and urothelium. Thus, sings of ureteral and bladder involvement by the disease may be nicely depicted [3]. The most useful radiologic feature of urinary tract tuberculosis is the multiplicity of abnormal findings [2], therefore the diagnosis of the disease can be suggested by a single examination (CT urography). Although MRI is reportedly of limited value in diagnostic images, it has an ability to depict the characteristic features corresponding to the subtle pathological changes and has some advantages including no radiation burden, detective capability of dilated collecting system, calyx and ureteral strictures independent of renal function $[3,7]$. In present case, these findings of local parenchymal thinning and dilated calices might be pathologically ascribed to characteristic tubercular changes, not only inflammation, fibrosis and destructive caseous necrosis in the renal parenchyma, but the stricture by fibrosis at ureter and/or calyx infundibula. Furthermore, the nodule in the renal parenchyma had isointensity on T1-weighted images and low intensity on T2-weighted images without gadolinium enhancement. This nodule seemed to correspond to
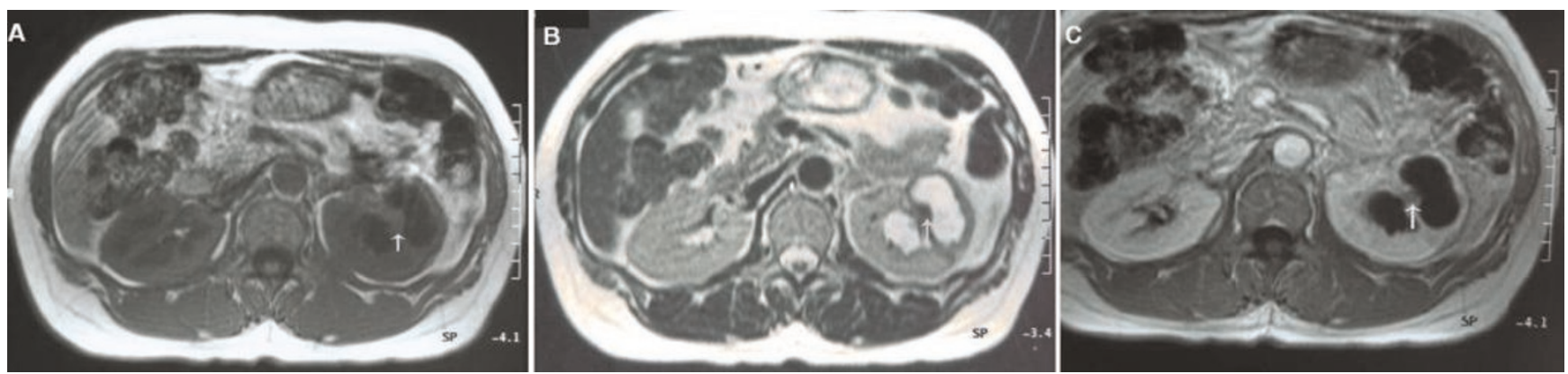

Figure 2. Transverse TI-weighted (A), T2-weighted (B) and Gadolinium (Gd) -enhanced TI-weighted (C) images are presented. Left kidney has cystic mass lesion at upper pole, which is composed of thinned parenchyma and water signal component. The abnormal renal parenchyma is demonstrated with low signal intensity on both TI and T2-weighted images. A small-sized parenchymal nodule (arrow) is also depicted isointense and hypointense on TI and T2-weighted images, respectively, when compared to the normal renal parenchyma. The abnormal parenchyma is heterogeneously enhanced and the nodule is not enhancing after Gd administration. 
a caseous necrotic granuloma found in the resected specimen. Thus MRI provided us a clue to further examinations in focusing on urinary tract tuberculosis leading to successful diagnosis. Although CT is indeed the most common modality in diagnostic images, the diagnosis cannot be made by a single examination and requires multi-modality methods [4]. Present case suggests that MRI could provide more informative features of renal tuberculosis, especially in case of no calcifications.

\section{Abbreviations}

CT, computed tomography; MRI, magnetic resonance imaging; PCR, polymerase chain reaction.

\section{Consent}

Written informed consent was obtained from the patient for publication of this report and accompanying images. A copy of the written consent is available for review by the Editor-in-Chief of this journal.

\section{Competing interests}

The authors declare that they have no competing interests.

\section{Authors' contributions}

NM was the primary person responsible for the writing of the manuscript. TM edited and coordinated the manuscript. All authors of this manuscript have read and approved the final version submitted.

\section{References}

I. Lenk S, Schroeder J: Genitourinary tuberculosis. Curr Opin Urol 200 I, I I:93-98

2. Gibson MS, Puckett ML, Shelly ME: Renal tuberculosis. Radiographics 2004, 24:25I-256.

3. Mapukata A, Andronikos S, Fasulakis S, McCulloch M, Grobbelaar M, Jee L: Modern imaging of renal tuberculosis in children. Australas Radiol 2007, 51:538-542.

4. Wang LJ, Wu CF, Wong YC, Chuang CK, Chu SH, Chen CJ: Imaging findings of urinary tuberculosis on excretory urogaphy and computerized tomography. J Urol 2003, 169:524-528.

5. Matos MJ, Bacelar MT, Pinto P, Ramos I: Genitourinary tuberculosis. Eur J Radiol 2005, 55: I8I- 187.

6. Verswijvel G, Janssens F, Vandevenne J, Stessens L, Meylaerts P, Palmers Y: Renal macronodular tuberculoma: $C T$ and MR findings in an asymptomatic patients. JBR-BTR 2002, 85:203-205.

7. Murata Y, Yamada I, Sumiya Y, Shichijo Y, Suzuki Y: Abdominal macronodular tuberculomas: MR findings. J Comput Assist Tomogr 1996, 20:643-646.

\section{Do you have a case to share?}

\section{Submit your case report today}

- Rapid peer review

- Fast publication

- PubMed indexing

- Inclusion in Cases Database

\section{Any patient, any case, can teach us something}

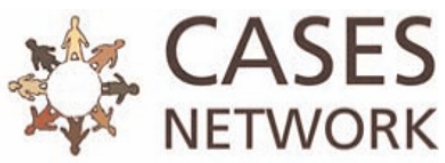

www.casesnetwork.com 